MSC 2010: 70E55, 70H09, 70H14

\title{
On Nonlinear Oscillations and Stability of Coupled Pendulums in the Case of a Multiple Resonance
}

\section{A. P. Markeev，T. N. Chekhovskaya}

The points of suspension of two identical pendulums moving in a homogeneous gravitational field are located on a horizontal beam performing harmonic oscillations of small amplitude along a fixed horizontal straight line passing through the points of suspension of the pendulums. The pendulums are connected to each other by a spring of low stiffness. It is assumed that the partial frequency of small oscillations of each pendulum is exactly equal to the frequency of horizontal oscillations of the beam. This implies that a multiple resonance occurs in this problem, when the frequency of external periodic action on the system is equal simultaneously to two its frequencies of small (linear) natural oscillations. This paper solves the nonlinear problem of the existence and stability of periodic motions of pendulums with a period equal to the period of oscillations of the beam. The study uses the classical methods due to Lyapunov and Poincaré, KAM (Kolmogorov, Arnold and Moser) theory, and algorithms of computer algebra.

The existence and uniqueness of the periodic motion of pendulums are shown, its analytic representation as a series is obtained, and its stability is investigated. For sufficiently small oscillation amplitudes of the beam, depending on the value of the dimensionless parameter which characterizes the stiffness of the spring connecting the pendulums, the found periodic motion is either Lyapunov unstable or stable for most (in the sense of Lebesgue measure) initial conditions or formally stable (stable in an arbitrarily large, but finite, nonlinear approximation).

Keywords: nonlinear oscillations, resonance, stability, canonical transformations

Received October 31, 2020

Accepted November 16, 2020

This work was carried out under the grant of the Russian Science Foundation (project No. 19-11-00116) at the Moscow Aviation Institute (National Research University) and at the Ishlinsky Institute for Problems in Mechanics of the Russian Academy of Science.

Anatoly P. Markeev

anat-markeev@mail.ru

Ishlinsky Institute for Problems in Mechanics RAS

prosp. Vernadskogo 101-1, Moscow, 119526 Russia

Moscow Aviation Institute (National Research University)

Volokolamskoe shosse 4, Moscow, 125080 Russia

Tatiana N. Chekhovskaya

tatshup@gmail.com

Moscow Aviation Institute (National Research University)

Volokolamskoe shosse 4, Moscow, 125080 Russia

RUSSIAN JOURNAL OF NONLINEAR DYNAMICS, 2020, 16(4), 607-623 


\section{Introduction}

Consider a system of two identical pendulums moving in a homogeneous gravitational field. Each of the pendulums is a weightless rod of length $\ell$. Point masses weighing $m g$ each are fastened to the ends $A_{1}$ and $A_{2}$ of these rods. The distance between the suspension points $O_{1}$ and $\mathrm{O}_{2}$ of the pendulums is constant and equal to $\delta$. The suspension points themselves are located on a horizontal beam which performs harmonic oscillations of small amplitude $a$ along a horizontal straight line (the point $O$ shown in Fig. 1 is a fixed point of this straight line). The pendulums are connected to each other by a linear elastic spring of low stiffness $k$. The distances $O_{1} B_{1}$ and $O_{2} B_{2}$ of the points of attachment of the spring to the pendulums from their suspension points are the same and equal to $b$. In an unstressed state the length of the spring is equal to the distance $\delta$ between the suspension points. It is assumed that the oscillation frequency $\omega$ of the beam is exactly equal to the partial frequency $\sqrt{g / \ell}$ of small linear oscillations of each pendulum. The motion of the pendulums occurs in a fixed vertical plane passing through points $O_{1}$ and $O_{2}$.

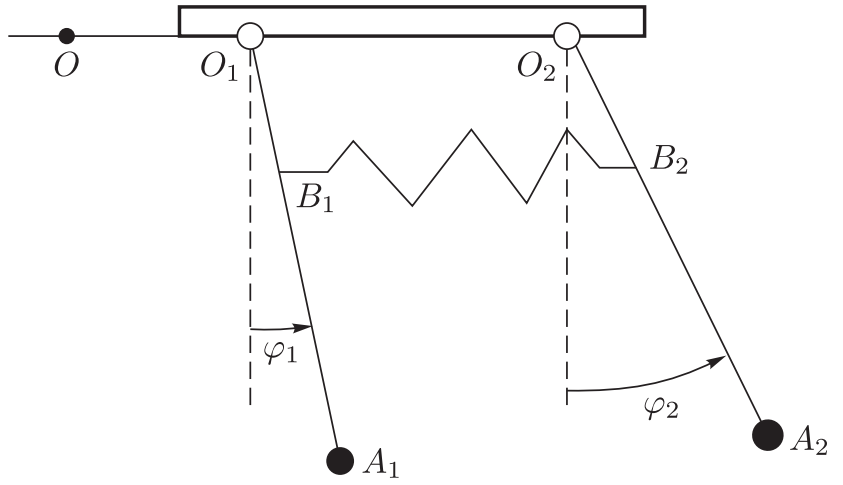

Fig. 1. Coupled pendulums suspended from a beam that oscillates along a horizontal straight line $\left(O_{1} A_{1}=O_{2} A_{2}=\ell, O_{1} B_{1}=O_{2} B_{2}=b, O_{1} O_{2}=\delta, O O_{1}=a \cos \omega t, \omega=\sqrt{g / \ell}\right)$.

Let $\varphi_{1}$ and $\varphi_{2}$ be the angles made by the rods $O_{1} A_{1}$ and $O_{2} A_{2}$ with the vertical. For the kinetic and potential energies of the system of two pendulums we have the following expressions (the dot denotes differentiation with respect to time $t$ ):

$$
\begin{gathered}
T=T_{2}+T_{1}+T_{0}, \quad \Pi=-m g \ell\left(\cos \varphi_{1}+\cos \varphi_{2}\right)+\frac{1}{2} k \Delta^{2}, \\
T_{2}=\frac{1}{2} m \ell^{2}\left(\dot{\varphi}_{1}^{2}+\dot{\varphi}_{2}^{2}\right), \quad T_{1}=-m a \ell \omega \sin \omega t\left(\cos \varphi_{1} \dot{\varphi_{1}}+\cos \varphi_{2} \dot{\varphi_{2}}\right), \\
T_{0}=m \omega^{2} a^{2} \sin ^{2} \omega t, \\
\Delta=\sqrt{\left[b\left(\cos \varphi_{2}-\cos \varphi_{1}\right)\right]^{2}+\left[\delta+b\left(\sin \varphi_{2}-\sin \varphi_{1}\right)\right]^{2}}-\delta .
\end{gathered}
$$

The system of differential equations given by the Lagrangian function $L=T-\Pi$ admits, for any parameter values, a family of partial solutions in which, for any $t$, the pendulums deviate from the vertical by equal angles $\varphi_{1} \equiv \varphi_{2}=\varphi(t)$, where $\varphi(t)$ satisfies the second-order differential equation:

$$
\frac{d^{2} \varphi}{d t^{2}}+\frac{g}{\ell} \sin \varphi=\frac{a}{\ell} \omega^{2} \cos \omega t \cos \varphi
$$

This equation describes the motion of a mathematical pendulum in the presence of harmonic horizontal oscillations of its suspension point with amplitude $a$ and frequency $\omega$. 
The case $\omega=\sqrt{g / \ell}$ studied in this paper corresponds to the multiple $1: 1: 1$ resonance, which has the property that the frequency of external periodic action on the system coincides with each of its two frequencies of small natural oscillations.

Nonlinear resonant oscillations of coupled pendulums without external periodic actions were investigated earlier in [1-3]. Forced oscillations at the $1: 1: 1$ resonance were considered in the report [4], which contains an analysis of the problem of the existence and linear stability of possible types of periodic motions. Below we consider one of these motions, namely, that for which $\varphi_{1}(t) \equiv \varphi_{2}(t)$. A procedure for constructing periodic motion is presented, its analytic representation is obtained and the nonlinear problem of stability is considered. The investigation relies on the classical and modern methods of nonlinear dynamics [5-11].

A systematic study of the problem of nonlinear oscillations of Hamiltonian systems in the presence of resonance goes back to the turn of the 20th century [12-14]. A short bibliography of works on the theory and applications of resonant problems in mechanics and physics can be found, for example, in [15]. References [16-19] address several model problems which are described by an autonomous Hamiltonian system at the 1:1 resonance and are important for astrophysics and celestial mechanics. In $[15,20]$ the $1: 1$ resonance was considered in the problem of the motion of a satellite, a rigid body, relative to the center of mass in a circular orbit. Nonlinear oscillations of a dynamically symmetric satellite at the $1: 1: 1$ resonance in an elliptic orbit of small eccentricity were examined in [21-23]. The problem of the stability of an autonomous Hamiltonian system in the degenerate (transcendental) resonant case was studied in [24]. Multiple resonances, different from the $1: 1: 1$ resonance, in a nonautonomous Hamiltonian system with two degrees of freedom were investigated in the recent series of publications [25-32]. General theoretical results obtained in these works were applied in investigating the motion of a satellite, a rigid body, relative to the center of mass and in analyzing the stability of Lagrangian solutions to the planar bounded elliptic three-body problem.

\section{The Hamiltonian function and its expansion as a series}

As generalized momenta corresponding to generalized coordinates $\varphi_{i}$, we take the dimensionless variables $p_{\varphi_{i}}$ defined by the equations

$$
p_{\varphi_{i}}=\frac{1}{m \ell \sqrt{g \ell}} \frac{\partial L}{\partial \dot{\varphi}_{i}} \quad(i=1,2) .
$$

From these equations we obtain expressions for generalized velocities:

$$
\dot{\varphi}_{i}=\omega\left(p_{\varphi_{i}}+\frac{a}{\ell} \sin \omega t \cos \varphi_{i}\right) \quad(i=1,2)
$$

Instead of time $t$, as an independent variable we take the dimensionless variable

$$
\tau=\omega t
$$

The canonically conjugate variables $\varphi_{i}, p_{\varphi_{i}}(i=1,2)$ correspond to the Hamiltonian function $H^{\prime}$ which is calculated from the formula

$$
H^{\prime}=\frac{1}{m g \ell}\left(T_{2}-T_{0}+\Pi\right)
$$


on the right-hand side of which the variables $\dot{\varphi}_{i}$ should be replaced with their expressions (2.1). This yields

$$
\begin{gathered}
H^{\prime}=\sum_{i=1}^{2}\left[\frac{1}{2} p_{\varphi_{i}}^{2}-\cos \varphi_{i}+\frac{a}{\ell} \sin \tau \cos \varphi_{i} p_{\varphi_{i}}+\frac{1}{2}\left(\frac{a}{\ell}\right)^{2} \sin ^{2} \tau \cos ^{2} \varphi_{i}\right]+ \\
+\frac{1}{2} \beta\left\{\sqrt{\left(\cos \varphi_{2}-\cos \varphi_{1}\right)^{2}+\left[\gamma+\left(\sin \varphi_{2}-\sin \varphi_{1}\right)\right]^{2}}-\gamma\right\}^{2} \\
\beta=\frac{k b^{2}}{m g l}, \quad \gamma=\frac{\delta}{b} .
\end{gathered}
$$

For ease of the calculations presented below to investigate the motions in which $\varphi_{1}=\varphi_{2}$, it is worthwhile to make a canonical transformation (with a valence equal to $1 / 2$ ) $\varphi_{i}, p_{\varphi_{i}} \rightarrow \widetilde{\varphi}_{i}, \widetilde{p}_{\varphi_{i}}$ using the formulae

$$
\begin{array}{cc}
\widetilde{\varphi}_{1}=\frac{1}{2}\left(\varphi_{1}+\varphi_{2}\right), & \widetilde{\varphi}_{2}=\frac{1}{2}\left(\varphi_{1}-\varphi_{2}\right), \\
\widetilde{p}_{\varphi_{1}}=\frac{1}{2}\left(p_{\varphi_{1}}+p_{\varphi_{2}}\right), & \widetilde{p}_{\varphi_{2}}=\frac{1}{2}\left(p_{\varphi_{1}}-p_{\varphi_{2}}\right) .
\end{array}
$$

The new variables correspond to a Hamiltonian function of the form

$$
\begin{aligned}
\widetilde{H} & =\frac{1}{2}\left(\widetilde{p}_{\varphi_{1}}^{2}+\widetilde{p}_{\varphi_{2}}^{2}\right)-\cos \widetilde{\varphi}_{1} \cos \widetilde{\varphi}_{2}+ \\
& +\frac{1}{4} \beta\left[\sqrt{4 \sin ^{2} \widetilde{\varphi}_{1} \sin ^{2} \widetilde{\varphi}_{2}+\left(\gamma-2 \cos \widetilde{\varphi}_{1} \sin \widetilde{\varphi}_{2}\right)^{2}}-\gamma\right]^{2}+ \\
& +\frac{a}{\ell} \sin \tau\left(\widetilde{p}_{\varphi_{1}} \cos \widetilde{\varphi}_{1} \cos \widetilde{\varphi}_{2}-\widetilde{p}_{\varphi_{2}} \sin \widetilde{\varphi}_{1} \sin \widetilde{\varphi}_{2}\right)+\frac{1}{4}\left(\frac{a}{\ell}\right)^{2} \sin ^{2} \tau \cos 2 \widetilde{\varphi}_{1} \cos 2 \widetilde{\varphi}_{2} .
\end{aligned}
$$

The motions under study (for which $\varphi_{1}(t) \equiv \varphi_{2}(t)$ ) are given by the equation $\widetilde{\varphi}_{2}=\widetilde{p}_{\varphi_{2}}=0$, and $\widetilde{\varphi}_{1}(\omega t)$ is a solution to Eq. (1.2).

Let us introduce a small parameter $\varepsilon$ by setting

$$
a=\varepsilon^{3} \ell, \quad \beta=\varepsilon^{2} s \quad(0<\varepsilon \ll 1) .
$$

and make a canonical transformation $\widetilde{\varphi}_{i}, \widetilde{p}_{\varphi_{i}} \rightarrow Q_{i}, P_{i}$ (with a valence equal to $\varepsilon^{-2}$ ):

$$
\widetilde{\varphi}_{1}=\varepsilon Q_{1}, \quad \widetilde{\varphi}_{2}=\varepsilon Q_{2}, \quad \widetilde{p}_{\varphi_{1}}=\varepsilon P_{1}, \quad \widetilde{p}_{\varphi_{2}}=\varepsilon P_{2} .
$$

The new Hamiltonian function $G$ can be represented by a convergent series in powers of $\varepsilon$ (the terms that do not depend on $Q_{1}, Q_{2}, P_{1}, P_{2}$ have been discarded):

$$
G=G_{0}+\varepsilon G_{1}+\varepsilon^{2} G_{2}+\varepsilon^{3} G_{3}+\varepsilon^{4} G_{4}+\varepsilon^{5} G_{5}+\ldots
$$

The coefficients of this series are polynomials in $Q_{1}, Q_{2}, P_{1}, P_{2}$ and are $2 \pi$-periodic in $\tau$. For the first six of them one can obtain the following expressions:

$$
\begin{gathered}
G_{0}=\frac{1}{2} \sum_{i=1}^{2}\left(Q_{i}^{2}+P_{i}^{2}\right), \quad G_{1}=0, \\
G_{2}=-\frac{1}{24} Q_{1}^{4}-\frac{1}{4} Q_{1}^{2} Q_{2}^{2}-\frac{1}{24} Q_{2}^{4}+\sin \tau P_{1}+s Q_{2}^{2}, \quad G_{3}=0, \\
G_{4}=\frac{1}{720}\left(Q_{1}^{6}+Q_{2}^{6}\right)+\frac{1}{48} Q_{1}^{2} Q_{2}^{2}\left(Q_{1}^{2}+Q_{2}^{2}\right)-\frac{1}{2} \sin \tau\left[P_{1}\left(Q_{1}^{2}+Q_{2}^{2}\right)+2 Q_{1} Q_{2} P_{2}\right]- \\
-\frac{1}{3} s Q_{2}^{2}\left(3 Q_{1}^{2}+Q_{2}^{2}\right), \quad G_{5}=-\frac{2 s}{\gamma} Q_{1}^{2} Q_{2}^{3} .
\end{gathered}
$$


The motion under study corresponds to the periodic solution $Q_{i}=Q_{i}^{*}(\tau), P_{i}=P_{i}^{*}(\tau)(i=1,2)$ of canonical equations with the Hamiltonian function (2.10). In this case, $Q_{2}^{*}=P_{2}^{*} \equiv 0$ and $Q_{1}^{*}$ satisfies the second-order differential equation

$$
\frac{d^{2} Q_{1}^{*}}{d \tau^{2}}+Q_{1}^{*}=\varepsilon^{2}\left(\cos \tau+\frac{1}{6} Q_{1}^{* 3}\right)-\varepsilon^{4}\left(\frac{1}{2} \cos \tau Q_{1}^{* 2}+\frac{1}{120} Q_{1}^{* 5}\right)+O\left(\varepsilon^{6}\right) .
$$

If the function $Q_{1}^{*}$ is found, then $P_{1}^{*}$ is calculated according to the equation

$$
P_{1}^{*}=\frac{d Q_{1}^{*}}{d \tau}-\varepsilon^{2} \sin \tau+O\left(\varepsilon^{4}\right)
$$

\section{Construction of a periodic solution}

The periodic solution $Q_{1}^{*}(\tau)$ to Eq. (2.14) can be found using an algorithm based on the Poincaré method for quasi-linear systems with one degree of freedom [6]. Set

$$
Q_{1}^{*}=c_{1} \sin \tau+c_{2} \cos \tau+\varepsilon^{2} f_{2}(\tau)+\varepsilon^{4} f_{4}(\tau)+\ldots,
$$

where $c_{1}, c_{2}$ are constants and $f_{2}, f_{4}, \ldots$ are $2 \pi$ - periodic functions which need to be found. Substituting this expression for $Q_{1}^{*}$ into Eq. (2.14) and equating the coefficients at $\varepsilon^{2}$ and $\varepsilon^{4}$ in series expansions of its left- and right-hand sides, we obtain

$$
\begin{aligned}
& \frac{d^{2} f_{2}}{d \tau^{2}}+f_{2}=\cos \tau+\frac{1}{6}\left(c_{1} \sin \tau+c_{2} \cos \tau\right)^{3} \\
& \frac{d^{2} f_{4}}{d \tau^{2}}+f_{4}=-\frac{1}{2}\left(c_{1} \sin \tau+c_{2} \cos \tau\right)^{2}\left[\cos \tau-f_{2}+\frac{1}{60}\left(c_{1} \sin \tau+c_{2} \cos \tau\right)^{3}\right] .
\end{aligned}
$$

In order for Eq. (3.2) to admit a periodic solution, it is necessary and sufficient that the coefficients at $\sin \tau$ and $\cos \tau$ on its right-hand side be equal. This condition allows a unique definition of the constants $c_{1}$ and $c_{2}$. We find

$$
c_{1}=0, \quad c_{2}=-2
$$

For this choice of $c_{1}$ and $c_{2}$ the system of equations (3.2)-(3.3) can be written as

$$
\begin{aligned}
& \frac{d^{2} f_{2}}{d \tau^{2}}+f_{2}=-\frac{1}{3} \cos 3 \tau \\
& \frac{d^{2} f_{4}}{d \tau^{2}}+f_{4}=2 \cos ^{2} \tau\left(f_{2}-\cos \tau+\frac{2}{15} \cos ^{3} \tau\right)
\end{aligned}
$$

From (3.5) we have

$$
f_{2}=c_{3} \sin \tau+c_{4} \cos \tau+\frac{1}{24} \cos 3 \tau,
$$

where $c_{3}$ and $c_{4}$ are constants. For such a function $f_{2}$ the right-hand side of Eq. (3.6) can be written as

$$
\frac{1}{80}\left[40 c_{3} \sin \tau+\left(120 c_{4}-105\right) \cos \tau+A_{3} \sin 3 \tau+B_{3} \cos 3 \tau+A_{5} \sin 5 \tau+B_{5} \cos 5 \tau\right] .
$$


We do not present the expressions for the constant coefficients $A_{3}, B_{3}, A_{5}, B_{5}$ since they will not be required in what follows. From the condition that there are no harmonics $\sin \tau$ and $\cos \tau$ in the last expression, we obtain

$$
c_{3}=0, \quad c_{4}=\frac{7}{8} .
$$

From (3.1), (3.4), (3.7) and (3.10) we have the following expression for the periodic solution $Q_{1}^{*}(\tau)$ to equation (2.14) calculated with an error of order $\varepsilon^{4}$ :

$$
Q_{1}^{*}=-2 \cos \tau+\frac{1}{24} \varepsilon^{2}(21 \cos \tau+\cos 3 \tau)+O\left(\varepsilon^{4}\right) .
$$

The function $P_{1}^{*}(\tau)$ corresponding to this solution is found from (2.15) and (3.9):

$$
P_{1}^{*}=2 \sin \tau-\frac{1}{8} \varepsilon^{2}(15 \sin \tau+\sin 3 \tau)+O\left(\varepsilon^{4}\right) .
$$

We note that in the terms through degree three in $\varepsilon$ the constructed periodic solution does not depend on the parameter $\gamma$ contained in the fifth-degree terms of the expansion of the Hamiltonian function (2.10).

\section{The Hamiltonian function of the perturbed motion and its preliminary transformation}

To investigate the stability of the pendulums' motion described by the found periodic solution $Q_{1}=Q_{1}^{*}(\tau), P_{1}=P_{1}^{*}(\tau), Q_{2}=0, P_{2}=0$, we introduce perturbations $q_{j}, p_{j}(j=1,2)$ by setting

$$
Q_{1}=Q_{1}^{*}+q_{1}, \quad Q_{2}=q_{2}, \quad P_{1}=P_{1}^{*}+p_{1}, \quad P_{2}=p_{2} .
$$

The equations of the perturbed motion are given by the Hamiltonian function $\Phi$, which is calculated by the formula

$$
\Phi=G+q_{1} \frac{d P_{1}^{*}}{d \tau}-p_{1} \frac{d Q_{1}^{*}}{d \tau}
$$

where $G$ is the function (2.10), in which $Q_{j}, P_{j}(j=1,2)$ have been replaced by their expressions (4.1).

The function $\Phi$ is analytic in the variables $q_{j}, p_{j}(j=1,2)$ and $2 \pi$-periodic in $\tau$. Its expansion as a series contains no first-degree terms in $q_{j}, p_{j}(j=1,2)$. Neglecting terms that are insignificant for the equations of motion and do not depend on $q_{j}, p_{j}(j=1,2)$, one can write this expansion as

$$
\begin{aligned}
\Phi & =\Phi_{2}+\Phi_{3}+\Phi_{4}+O\left(\left(q_{1}^{2}+p_{1}^{2}+q_{2}^{2}+p_{2}^{2}\right)^{5 / 2}\right), \\
\Phi_{2} & =\frac{1}{2}\left(q_{1}^{2}+p_{1}^{2}+q_{2}^{2}+p_{2}^{2}\right)+\varepsilon^{2}\left[-\cos ^{2} \tau q_{1}^{2}+\left(s-\cos ^{2} \tau\right) q_{2}^{2}\right]+\varepsilon^{4} \Phi_{2}^{*}, \\
\Phi_{3} & =\varepsilon^{2}\left(\frac{1}{3} \cos \tau q_{1}^{3}+\cos \tau q_{1} q_{2}^{2}\right)+\varepsilon^{4} \Phi_{3}^{*}, \\
\Phi_{4} & =\varepsilon^{2}\left[-\frac{1}{24}\left(q_{1}^{4}+q_{2}^{4}\right)-\frac{1}{4} q_{1}^{2} q_{2}^{2}\right]+\varepsilon^{4} \Phi_{4}^{*} .
\end{aligned}
$$

Here $\Phi_{k}^{*}(k=2,3,4)$ is the form of degree $k$ in $q_{1}, q_{2}, p_{1}, p_{2}$ with coefficients $2 \pi$-periodic in $\tau$. 
For further analysis it is worthwhile to make the transformation $q_{j}, p_{j} \rightarrow U_{j}, V_{j}(j=1,2)$, which eliminates in the form (4.4) its first four terms independent of the parameter $\varepsilon$. This transformation is a rotation by angle $\tau$ in the planes $q_{1} p_{1}$ and $q_{2} p_{2}$, is canonical and given by

$$
q_{j}=U_{j} \cos \tau+V_{j} \sin \tau, \quad p_{j}=-U_{j} \sin \tau+V_{j} \cos \tau \quad(j=1,2) .
$$

The new Hamiltonian function $F$ is obtained from the function (4.3) by discarding the first four terms from the form $\Phi_{2}$ and by subsequently replacing $q_{j}, p_{j}$ with their expressions (4.7). The function $F$ is of order two in $\varepsilon$.

The function $F$ can be simplified by making a canonical, near-identity change of variables $U_{j}, V_{j} \rightarrow u_{j}, v_{j}$ which eliminates the independent variable $\tau$ from the terms of order $\varepsilon^{2}$ in the expansion of $F$ as a series in powers of $\varepsilon$. The explicit form of the change of variables $U_{j}, V_{j} \rightarrow u_{j}, v_{j}$ will not be required in what follows and is therefore not presented. The new variables $u_{j}, v_{j}$ correspond to the Hamiltonian function $\Gamma$ of the form

$$
\begin{aligned}
\Gamma & =\Gamma_{2}+\Gamma_{3}+\Gamma_{4}+O\left(\left(u_{1}^{2}+v_{1}^{2}+u_{2}^{2}+v_{2}^{2}\right)^{5 / 2}\right), \\
\Gamma_{2} & =\varepsilon^{2}\left(-\frac{3}{8} u_{1}^{2}-\frac{1}{8} v_{1}^{2}+\frac{4 s-3}{8} u_{2}^{2}+\frac{4 s-1}{8} v_{2}^{2}\right)+\varepsilon^{4} \Gamma_{2}^{*}, \\
\Gamma_{3} & =\varepsilon^{2}\left(\frac{3}{8} u_{2}^{2} u_{1}+\frac{1}{8} v_{2}^{2} u_{1}+\frac{1}{4} u_{2} v_{2} v_{1}+\frac{1}{8} u_{1} v_{1}^{2}+\frac{1}{8} u_{1}^{3}\right)+\varepsilon^{4} \Gamma_{3}^{*}, \\
\Gamma_{4} & =\varepsilon^{2}\left[-\frac{1}{32}\left(u_{1}^{2} v_{1}^{2}+3 u_{1}^{2} u_{2}^{2}+u_{1}^{2} v_{2}^{2}+u_{2}^{2} v_{1}^{2}+3 v_{1}^{2} v_{2}^{2}+u_{2}^{2} v_{2}^{2}\right)-\right. \\
& \left.-\frac{1}{64}\left(u_{1}^{4}+u_{2}^{4}+v_{1}^{4}+v_{2}^{4}\right)-\frac{1}{8} u_{1} u_{2} v_{1} v_{2}\right]+\varepsilon^{4} \Gamma_{4}^{*},
\end{aligned}
$$

where $\Gamma_{k}^{*}(k=2,3,4)$ is the form of degree $k$ in $u_{1}, u_{2}, v_{1}, v_{2}$ with coefficients $2 \pi$-periodic in $\tau$. Note that $\Gamma_{k}-\varepsilon^{4} \Gamma_{k}^{*}(k=2,3,4)$ are averaged (over $\tau$ ) sets of terms of order $\varepsilon^{2}$ in the functions (4.4)-(4.6), in which the transformation (4.7) was made and then $U_{j}, V_{j}$ were replaced with $u_{j}, v_{j}$.

\section{Analysis of the linear problem}

The characteristic equation of the linearized system of equations of the perturbed motion is reciprocal, as is that of any linear system of Hamiltonian differential equations with periodic coefficients, and its roots (multipliers) are continuous in $\varepsilon[5,6]$. It immediately follows from this fact and from (4.9) that, if the parameters $(4 s-3)$ and $(4 s-1)$ have opposite signs, then at sufficiently small $\varepsilon$ the charaacteristic equation has a root whose absolute value is larger than 1 . Therefore [5], in the interval

$$
\frac{1}{4}<s<\frac{3}{4}
$$

the periodic motion of the pendulums is Lyapunov unstable (not only by virtue of the linearized equations of the perturbed motion, but also in a complete nonlinear system with the Hamiltonian function (4.8)).

If

$$
0 \leqslant s \leqslant \frac{1}{4} \quad \text { or } \quad s \geqslant \frac{3}{4}
$$

then, at small $\varepsilon$, all multipliers have absolute values equal to 1 , and a rigorous solution to the problem of stability requires an additional (nonlinear) analysis of the equations of the perturbed motion. 
For values of $s$ from the intervals (5.2), the characteristic exponents defined by linear equations with the Hamiltonian function (4.9) will be (for small $\varepsilon$ ) purely imaginary quantities $\pm i \lambda_{1}, \pm i \lambda_{2}$, where $i$ is an imaginary unit and $\lambda_{1}, \lambda_{2}$ are real numbers of any sign. If $\lambda_{1}, \lambda_{2}$ are such that

$$
k_{1} \lambda_{1}+k_{2} \lambda_{2}=N
$$

where $N, k_{1}, k_{2}$ are integer numbers such that $\left|k_{1}\right|+\left|k_{2}\right|=k$, then the system exhibits a resonance of order $k$. In the stability problem, the most important resonances are those of lower orders (through order four).

To investigate the nonlinear problem, we first need to make a canonical transformation reducing the quadratic part $\Gamma_{2}$ of expansion (4.8) to normal coordinates. For this we make a linear change of variables $u_{1}, u_{2}, v_{1}, v_{2} \rightarrow \xi_{1}, \xi_{2}, \eta_{1}, \eta_{2}$ by the formulae

$$
\begin{gathered}
u_{1}=-\alpha \xi_{1}, \quad u_{2}=-\beta \xi_{2}, \quad v_{1}=\alpha^{-1} \eta_{1}, \quad v_{2}=\beta^{-1} \eta_{2}, \\
\alpha=\frac{1}{\sqrt[4]{3}}, \quad \beta=\sqrt[4]{\frac{4 s-1}{4 s-3}} .
\end{gathered}
$$

This transformation is canonical with a valence equal to -1 . It transforms the function $\Gamma_{2}$ to a function $\widetilde{\Gamma}_{2}$ of the form

$$
\widetilde{\Gamma}_{2}=\varepsilon^{2}\left[\frac{1}{2} \omega_{1}\left(\xi_{1}^{2}+\eta_{1}^{2}\right)-\frac{1}{2} \sigma \omega_{2}\left(\xi_{2}^{2}+\eta_{2}^{2}\right)\right]+\varepsilon^{4} \widetilde{\Gamma}_{2}^{*},
$$

where

$$
\omega_{1}=\frac{\sqrt{3}}{4} \simeq 0.433013, \quad \omega_{2}=\frac{1}{4} \sqrt{(4 s-1)(4 s-3)},
$$

and $\sigma=-1$ in the region $0<s<1 / 4$ and $\sigma=1$ in the region $s>3 / 4$. In the interval $0<s<1 / 4$ the parameter $\omega_{2}$ decreases monotonically from the value $\sqrt{3} / 4$ to zero, and in the interval $s>3 / 4$ it increases monotonically. Graphs of the function $\omega_{2}(s)$ are presented in Figs. 2 and 3.

If we eliminate the value $s=1$ which lies in the region $s>3 / 4$ and for which $\omega_{1}=\omega_{2}=$ $=\sqrt{3} / 4$, then for the other values of $s$ inside the regions (5.2) of linear stability at small $\varepsilon$ no first- or second-order resonances are possible. For such parameter values, we use a linear real canonical transformation $\xi_{1}, \xi_{2}, \eta_{1}, \eta_{2} \rightarrow x_{1}, x_{2}, X_{1}, X_{2}, 2 \pi$-periodic in $\tau$ and analytic in $\varepsilon$, which can be constructed, for example, by the Deprit-Hori method [8, 33]. Then the Hamiltonian function of the perturbed motion reduces to the form

$$
H=H_{2}+H_{3}+H_{4}+O\left(\left(x_{1}^{2}+X_{1}^{2}+x_{2}^{2}+X_{2}^{2}\right)^{5 / 2}\right),
$$

where

$$
\begin{gathered}
H_{2}=\frac{1}{2} \lambda_{1}\left(x_{1}^{2}+X_{1}^{2}\right)+\frac{1}{2} \lambda_{2}\left(x_{2}^{2}+X_{2}^{2}\right), \\
\lambda_{1}=\varepsilon^{2}\left[\omega_{1}+O\left(\varepsilon^{2}\right)\right], \quad \lambda_{2}=\varepsilon^{2}\left[-\sigma \omega_{2}+O\left(\varepsilon^{2}\right)\right],
\end{gathered}
$$

and the third- and fourth-degree terms $H_{3}$ and $H_{4}$ in the expansion (5.7) are given by the equations

$$
H_{3}=\varepsilon^{2}\left[\frac{3^{3 / 4}}{24 \beta^{2}} x_{1} X_{2}^{2}+\frac{3^{1 / 4}}{24}\left(x_{1}^{3}+3 x_{1} X_{1}^{2}+6 x_{2} X_{1} X_{2}\right)+\frac{3^{3 / 4} \beta^{2}}{8} x_{1} x_{2}^{2}\right]+\varepsilon^{4} H_{3}^{*},
$$




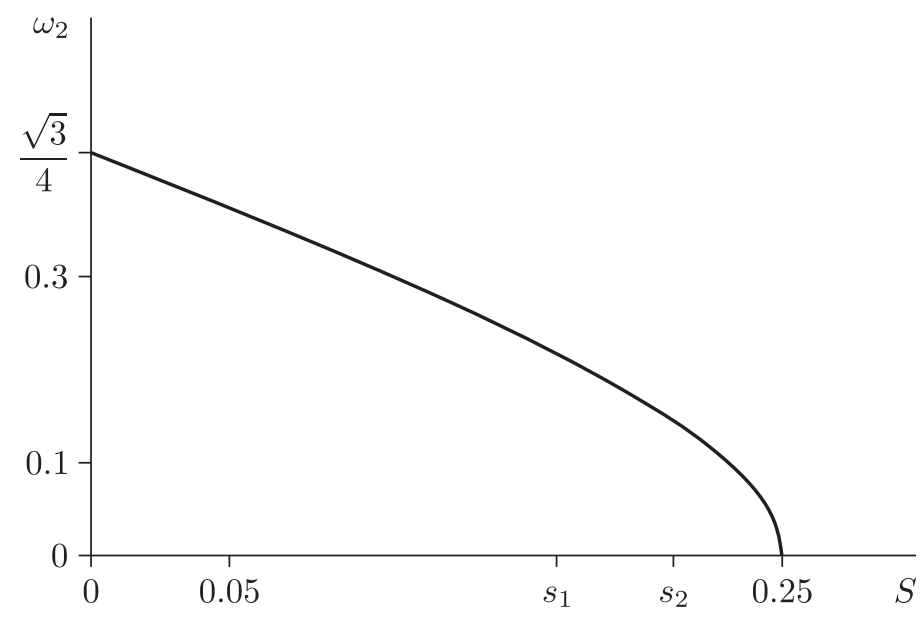

Fig. 2. Dependence of the frequency $\omega_{2}$ on $s$ at $0 \leqslant s \leqslant 1 / 4$.

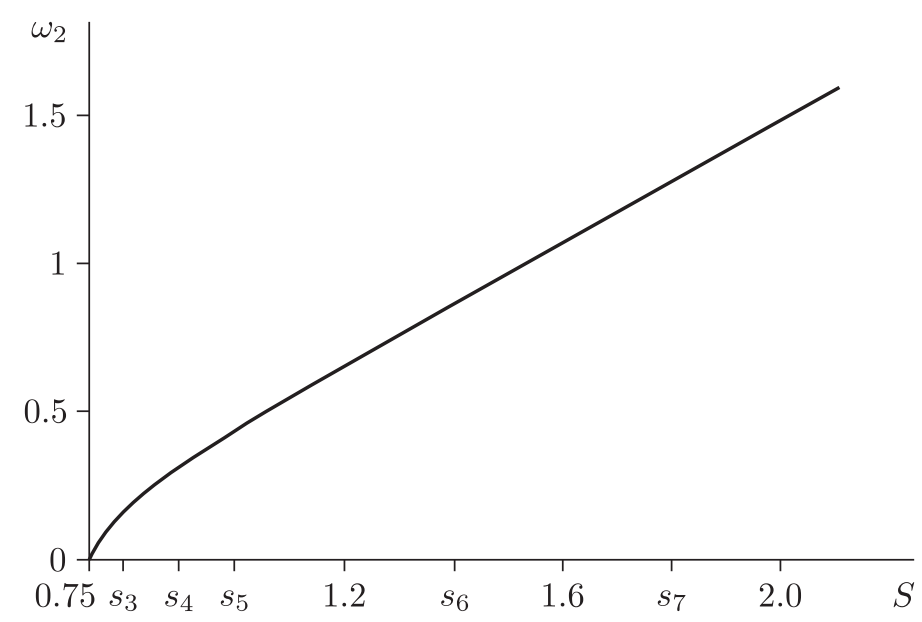

Fig. 3. Dependence of the frequency $\omega_{2}$ on $s$ at $s \geqslant 3 / 4$.

$$
\begin{aligned}
H_{4} & =\varepsilon^{2}\left[\frac{1}{64 \beta^{4}} X_{2}^{4}+\frac{\sqrt{3}}{96 \beta^{2}}\left(x_{1}^{2}+9 X_{1}^{2}\right) X_{2}^{2}+\frac{1}{192}\left(x_{1}^{4}+6 x_{1}^{2} X_{1}^{2}+24 x_{1} x_{2} X_{1} X_{2}+\right.\right. \\
& \left.\left.+9 X_{1}^{4}+6 x_{2}^{2} X_{2}^{2}\right)+\frac{\sqrt{3} \beta^{2}}{32}\left(x_{1}^{2}+X_{1}^{2}\right) x_{2}^{2}+\frac{\beta^{4}}{64} x_{2}^{4}\right]+\varepsilon^{4} H_{4}^{*} .
\end{aligned}
$$

Here, $H_{3}^{*}$ and $H_{4}^{*}$ are forms of degrees three and four in $x_{1}, x_{2}, X_{1}, X_{2}$ with coefficients $2 \pi$-periodic in $\tau$.

\section{On possible resonances}

Taking into account expressions (5.9) and (5.6) for $\lambda_{1}, \lambda_{2}$ and assuming that the dimensionless parameter $s$ (which characterizes the stiffness of the spring connecting the pendulums) cannot be arbitrarily large, we find that at small $\varepsilon$ the resonances (5.3) inside the regions (5.2) of linear stability take only place when the integer $N$ on the right-hand side of relation (5.3) is zero. If the order of the resonance does not exceed 4, then in the plane $s \varepsilon$ at small $\varepsilon$ there exist 
only seven resonant curves. In Figs. 2 and 3, they emanate from the corresponding generating points $\left(s_{i}, 0\right)$ of the abscissa axes.

In the region $0<s<1 / 4$, two resonances (see Fig. 2) are possible. For these resonances:
1) $\omega_{1}=2 \omega_{2}, s_{1}=\frac{1}{2}-\frac{1}{8} \sqrt{7} \simeq 0.169281$;
2) $\omega_{1}=3 \omega_{2}, s_{2}=\frac{1}{2}-\frac{1}{6} \sqrt{3} \simeq 0.211325$.

And in the region $s>3 / 4$ five resonances (Fig. 3) take place for which:
3) $\omega_{1}=3 \omega_{2}, s_{3}=\frac{1}{2}-\frac{1}{6} \sqrt{3} \simeq 0.788675$;
4) $\omega_{1}=2 \omega_{2}, s_{4}=\frac{1}{2}+\frac{1}{8} \sqrt{7} \simeq 0.830719$;
5) $\omega_{1}=\omega_{2}, s_{5}=1$;
6) $\omega_{2}=2 \omega_{1}, s_{6}=\frac{1}{2}+\frac{1}{4} \sqrt{13} \simeq 1.401388$;
7) $\omega_{2}=3 \omega_{1}, s_{7}=\frac{1}{2}+\frac{1}{2} \sqrt{7} \simeq 1.822876$.

The value of the parameter $s$ on the $i$ th resonant curve differs from its value $s_{i}$ at the generating point by values of order $\varepsilon^{2}$.

\section{A nonlinear problem inside the regions (5.2). The nonresonant case}

Let the parameter $s$ lie inside the regions (5.2) and suppose that its value is not resonant $\left(s \neq s_{i}(i=1,2, \ldots, 7)\right)$. Using a real near-identity $2 \pi$-periodic (in $\left.\tau\right)$ canonical Birkhoff transformation [7] $x_{1}, x_{2}, X_{1}, X_{2} \rightarrow y_{1}, y_{2}, Y_{1}, Y_{2}$, we reduce the Hamiltonian function (5.7) to its normal form through terms of degree four in $y_{1}, y_{2}, Y_{1}, Y_{2}$. In the symplectic polar coordinates $r_{j}, \psi_{j}$,

$$
y_{j}=\sqrt{2 r_{j}} \sin \psi_{j}, \quad Y_{j}=\sqrt{2 r_{j}} \cos \psi_{j} \quad(j=1,2)
$$

the normalized Hamiltonian function can be written as

$$
H=\lambda_{1} r_{1}+\lambda_{2} r_{2}+c_{20} r_{1}^{2}+c_{11} r_{1} r_{2}+c_{02} r_{2}^{2}+O\left(\left(r_{1}+r_{2}\right)^{5 / 2}\right),
$$

where $\lambda_{1}, \lambda_{2}$ are given by equations (5.9). But finding constant coefficients $c_{i j}$ requires rather cumbersome calculations. These calculations have been performed using methods of computer algebra, and the following expressions have been obtained for the coefficients $c_{i j}$ :

$$
\begin{gathered}
c_{20}=\varepsilon^{2}\left[-\frac{1}{96}+O\left(\varepsilon^{2}\right)\right], \quad c_{11}=\varepsilon^{2}\left[-\sigma \frac{\sqrt{3}(8 s-3)^{2}}{1536 \omega_{2}\left(\omega_{1}^{2}-4 \omega_{2}^{2}\right)}+O\left(\varepsilon^{2}\right)\right], \\
c_{02}=\varepsilon^{2}\left[\frac{(8 s-3)\left(1280 s^{3}-1376 s^{2}+264 s-9\right)}{24576 \omega_{2}^{2}\left(\omega_{1}^{2}-4 \omega_{2}^{2}\right)}+O\left(\varepsilon^{2}\right)\right] .
\end{gathered}
$$

In addition to considering the Lyapunov stability, we will also analyze the stability for most (in the sense of Lebesgue measure) initial conditions $u_{j}(0), v_{j}(0)(j=1,2)$ and the formal stability.

On the stability for most initial conditions (see [9, 34-37]). If the inequality

$$
c_{11}^{2}-4 c_{20} c_{02} \neq 0
$$

is satisfied, then most trajectories $u_{j}(\tau), v_{j}(\tau)(j=1,2)$ of the system with the Hamiltonian function (4.8) which satisfy the condition $u_{1}^{2}(0)+u_{2}^{2}(0)+v_{1}^{2}(0)+v_{2}^{2}(0)<\mu$ at the initial time 
$\tau=0$ remain for all $\tau \geqslant 0$ in a small neighborhood of the unperturbed motion $u_{j}=v_{j}=0$ $(j=1,2)$. The set of initial conditions corresponding to the above-mentioned "majority" has a relative measure that is not smaller than $1-O\left(\mu^{1 / 4}\right)$.

However, the trajectories starting in an addition to the set of "most" initial conditions which has a relative measure of order $\mu^{1 / 4}$ can, generally speaking, go arbitrarily far away from the initial position (this phenomenon is known as "Arnold diffusion"). But it was shown in [38-40] that for almost any system with a Hamiltonian function of the form (4.8) there exist positive numbers $\delta_{1}$ and $\delta_{2}$ such that the difference of the quantity $u_{1}^{2}(\tau)+u_{2}^{2}(\tau)+v_{1}^{2}(\tau)+v_{2}^{2}(\tau)$ from its small initial value does not exceed a quantity of order $\mu^{\delta_{1}}$ for all values of $\tau$ satisfying the inequality

$$
0<\tau<\exp \left(\mu^{-\delta_{2}}\right)
$$

This implies that, if there does exist "Arnold diffusion", it occurs exponentially slowly.

Based on the formulae (7.3) and (5.6), the left-hand side of inequality (7.4) can be rewritten as

$$
c_{11}^{2}-4 c_{20} c_{02}=\varepsilon^{4}\left[d+O\left(\varepsilon^{2}\right)\right],
$$

where the parameter $d$ does not depend on $\sigma$ and has the same expression in both regions $0<s<1 / 4$ and $s>3 / 4$ :

$$
d=-\frac{(8 s-3)\left(81920 s^{5}-169984 s^{4}+110336 s^{3}-22944 s^{2}+360 s+243\right)}{9437184 \omega_{2}^{2}\left(\omega_{1}^{2}-4 \omega_{2}^{2}\right)^{2}} .
$$

When $\varepsilon$ is small, the inequality $d \neq 0$ is $[9,37]$ a sufficient stability condition for most initial conditions.

1. Analysis shows that in the region $0<s<1 / 4$ the function $d(s)$ defined by equation (7.6) is positive for all values of $s$ (recall that the resonant values $s_{i}(i=1,2, \ldots, 7)$ are not considered in this section). Figure 4 shows a graph of the function $d(s)$ in the interval $1 / 8<s<1 / 4$. The curve $d(s)$ has two vertical asymptotes: $s=s_{1}$ (resonance $\omega_{1}=2 \omega_{2}$ ) and $s=1 / 4$ (the boundary of the region); the point $s=0.216774$ is a point of local minimum of the function $d(s)$, at this point $d=2.644152$. We also note that $d(0)=1 / 768 \simeq 0.001302$ (Fig. 4 does not show the point with coordinates 0 and $d(0))$.

Thus, if $s$ belongs to the interval $0<s<1 / 4$ (such that $s \neq s_{1}, s \neq s_{2}$ ), then for sufficiently small $\varepsilon$ the periodic motion of the pendulums is stable for most initial conditions.

2. The behavior of the function $d(s)$ with $s>3 / 4$ is more complex (see Figs. 5 and 6 ). The graph of the function $d(s)$ has two vertical asymptotes: $s=3 / 4$ (the boundary of the region) and $s=s_{4}$ (resonance $\omega_{1}=2 \omega_{2}$ ). In the interval $3 / 4<s<s_{4}$ the function $d(s)$ is positive and has the minimal value $d=0.441034$ at the point $s=0.770815$ (Fig. 5); when $s_{4}<s<1$, the function decreases monotonically from infinitely large values to the value $d(1)=115 / 186624 \simeq 0.000616$. When $1<s<s_{*}=1.004417$, the function $d(s)$ is positive, and when $s>s_{*}$, it is negative (Fig. 6); when $s=s_{*}$, the function $d(s)$ vanishes. The point $s=1.279761$ is a minimum point at which $d=-0.006942$. As $s$ increases, the graph of the function $d(s)$ approaches from below the horizontal asymptote $d=-5 / 1152 \simeq-0.004340$.

It follows from the analysis of the function $d(s)$ that in the region $s>3 / 4$ outside the resonant points $s_{i}(i=3, \ldots, 7)$ and the point $s_{*}$, at which $d=0$, stability takes place at small $\varepsilon$ for most initial conditions.

On the formal stability (see [41-48]). The concept of formal stability was introduced by J. Moser for systems possessing a sign-definite formal integral which is a (possibly divergent)

RUSSIAN JOURNAL OF NONLINEAR DYNAMICS, 2020, 16(4), 607-623 

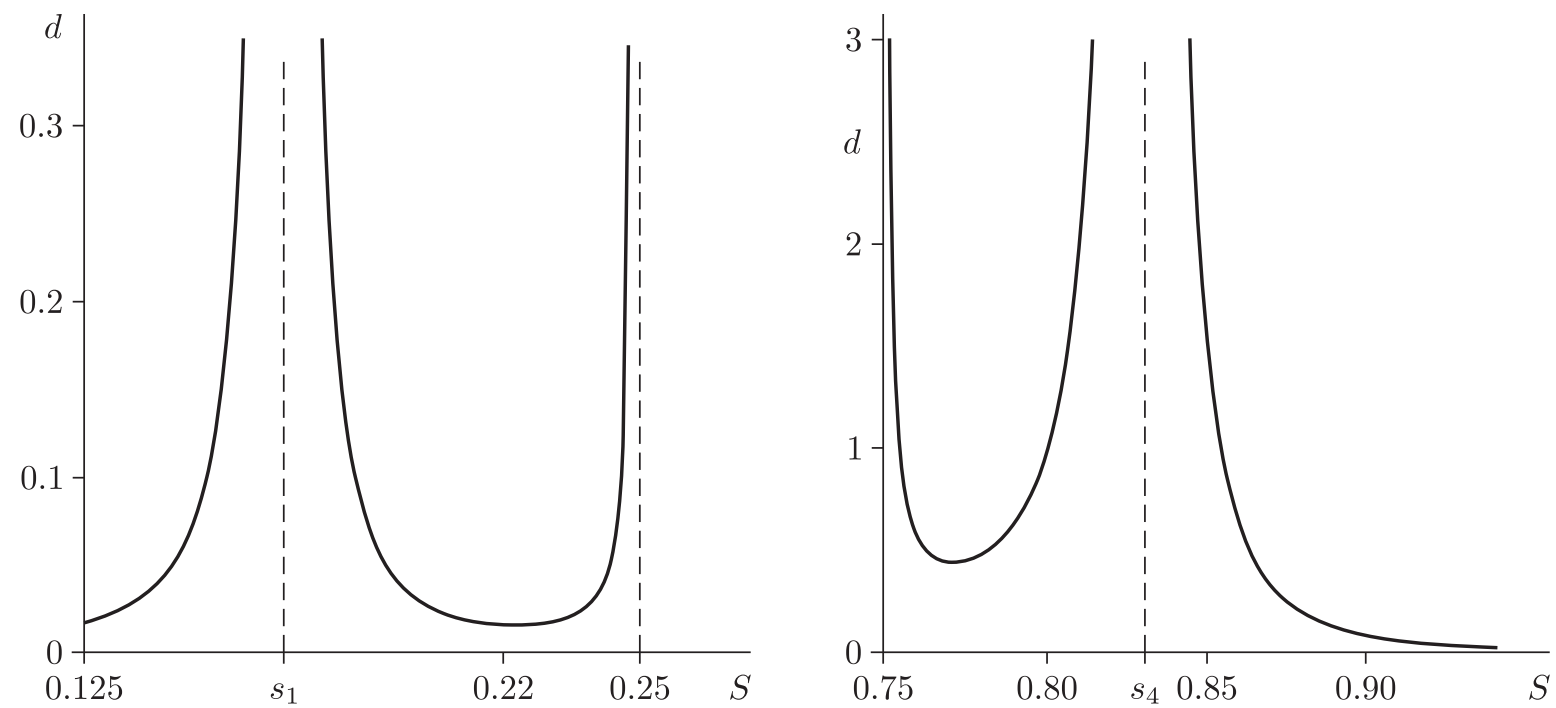

Fig. 4. Graph of the function $d(s)$ for $1 / 8<s<1 / 4$. Fig. 5. Graph of the function $d(s)$ for $3 / 4<s<1$.

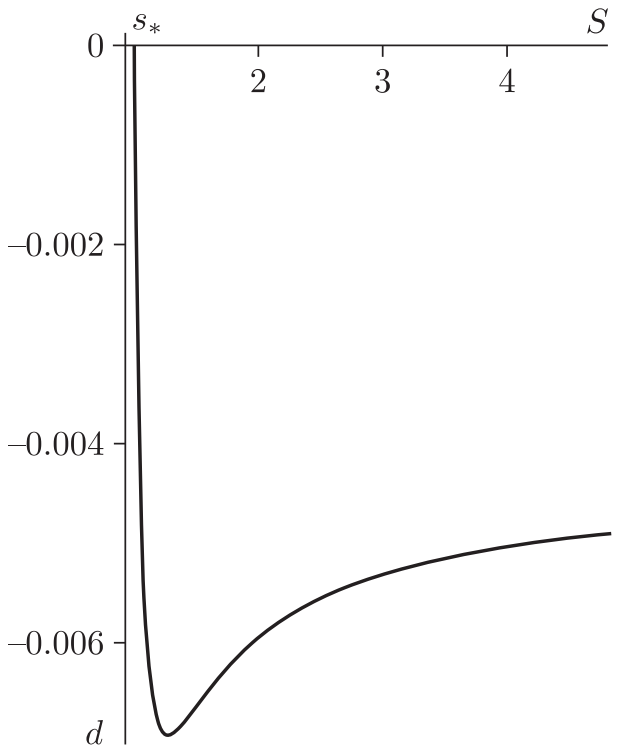

Fig. 6. Graph of the function $d(s)$ for $s>1 / 4$.

power series. This concept is very important in investigating the stability on a finite (but very large) time interval. The presence of formal stability implies that the Lyapunov instability (if there is any in the problem of interest) does not occur if terms up to an arbitrarily high (but finite) order in the coordinates and momenta of the perturbed motion are taken into account in the expansion of the Hamiltonian function. In the presence of formal stability, even if there exist trajectories going far away from the unperturbed motion, the motion along them is extremely slow. The corresponding estimates were obtained by J. Moser [42, 43], C. L. Siegel [45], J. Glimm [46], N. Nekhoroshev [39, 40] and others (see [9, 48]). Formal stability is quite sufficient to solve the question of stability in most physical and technical problems described by Hamiltonian differential equations. 
1. In the region $0<s<1 / 4$ the quantities $\lambda_{j}(j=1,2)$ from (5.9) at small $\varepsilon$ have the same signs (they are positive). Therefore [41], in this region, for all $s$ (including the resonant values $s_{1}$ and $s_{2}$ ) formal stability takes place if $\varepsilon$ is sufficiently small.

2. In contrast, in the region $s>3 / 4$ the quantities (5.9) with small $\varepsilon$ have different signs. Here, to solve the question of formal stability, we use the results of [47]. We assume that there are no resonances up to order four (i.e., $s \neq s_{i}, i=3, \ldots, 7$ ).

Consider the expression

$$
D=c_{20} \sigma_{2}^{2}+c_{11} \sigma_{1} \sigma_{2}+c_{02} \sigma_{1}^{2} \quad\left(\sigma_{j}=\left|\lambda_{j}\right|, j=1,2\right) .
$$

If the value of (7.7) does not vanish for $\sigma_{2}=r \sigma_{1}$, where $r$ is a rational number, then the unperturbed motion is formally stable [47].

Calculations show that, when $\sigma_{2}=r \sigma_{1}$, the expression for $D$ with an error of order $\varepsilon^{4}$ can be represented as

$$
D=\varepsilon^{2} \frac{243 P}{16384 \omega_{2}^{2}\left(3-64 \omega_{2}^{2}\right) Q},
$$

where $P$ and $Q$ are polynomials of the form

$$
\begin{aligned}
& P=16 r^{12}+184 r^{10}+577 r^{8}-1508 r^{6}+290 r^{4}+108 r^{2}-15 \\
& Q=8192 s^{6}-24576 s^{5}+38016 s^{4}-41984 s^{3}+28416 s^{2}-8928 s+783 .
\end{aligned}
$$

But it is easy to see that the polynomial $P$ with integer-valued coefficients has no rational roots $r=\ell / m$, where $\ell$ and $m$ are coprime integers. Indeed, otherwise (see, e.g., [49]) $\ell$ and $m$ must be the denominators of the numbers 15 and 16, respectively, and a simple enumeration of $\ell$ and $m$ shows that the numbers $r=\ell / m$ are not the roots of the polynomial $P(r)$.

Thus, for all $s>3 / 4$ (except perhaps for the values $s=s_{i}(i=3, \ldots, 7)$ which have not been considered so far) the motion under study is formally stable.

\section{A nonlinear problem in the region $s>3 / 4$. Resonant cases}

Resonance $\lambda_{1}+2 \lambda_{2}=0\left(s=s_{4}+O\left(\varepsilon^{2}\right)\right)$. Using the Birkhoff transformation, the Hamiltonian function (5.7) can be reduced to the following normal form:

$$
H=\lambda_{1} r_{1}+\lambda_{2} r_{2}+\chi \sqrt{r_{1}} r_{2} \sin \left(\psi_{1}+2 \psi_{2}\right)+O\left(\left(r_{1}+r_{2}\right)^{2}\right)
$$

where

$$
\chi=\varepsilon^{2} \frac{(1+\sqrt{7})}{212^{3 / 4}}+O\left(\varepsilon^{4}\right)
$$

For small $\varepsilon$ the parameter $\chi$ is nonzero. Therefore [11], instability takes place.

Resonance $2 \lambda_{1}+\lambda_{2}=0\left(s=s_{6}+O\left(\varepsilon^{2}\right)\right)$. Since the variables $x_{2}, X_{2}$ appear in the term $H_{3}$ of expansion (5.7) in powers whose sum is equal to two, the form $H_{3}$ contains no resonant terms and can be completely eliminated using the Birkhoff transformation. The Hamiltonian function normalized through terms of degree four in $y_{1}, y_{2}, Y_{1}, Y_{2}$ will be given by an equation of the form (7.2) in which

$$
\begin{gathered}
c_{20}=\varepsilon^{2}\left[-\frac{1}{96}+O\left(\varepsilon^{2}\right)\right], \quad c_{11}=\varepsilon^{2}\left[\frac{53+4 \sqrt{13}}{2160}+O\left(\varepsilon^{2}\right)\right], \\
c_{02}=\varepsilon^{2}\left[-\frac{2051+328 \sqrt{13}}{17280}+O\left(\varepsilon^{2}\right)\right] .
\end{gathered}
$$


For the left-hand side of inequality (7.4) we obtain the expression (7.5), in which

$$
d=-\frac{80227+13064 \sqrt{13}}{18662400} \simeq-0.006823 .
$$

It follows from (7.4), (8.2) and (8.3) that for sufficiently small $\varepsilon$ the form, quadratic (in $r_{1}, r_{2}$ ), in equation (7.2) will be sign-definite. Therefore [46], the motion under study is formally stable. It will also be stable for most initial conditions, since inequality (7.4) is obviously satisfied for small $\varepsilon$.

Resonances $\lambda_{1}+3 \lambda_{2}=0\left(s=s_{3}+O\left(\varepsilon^{2}\right)\right)$ and $3 \lambda_{1}+\lambda_{2}=0\left(s=s_{7}+O\left(\varepsilon^{2}\right)\right)$. These four-order resonances are investigated similarly to the above-mentioned third-order resonance $2 \lambda_{1}+\lambda_{2}=0$. Here one can also completely eliminate the third-degree terms $H_{3}$ in (5.7) and, similarly to the third-order resonance, the presence of commensurabilities $\lambda_{1}+3 \lambda_{2}=0$ and $3 \lambda_{1}+\lambda_{2}$ does not lead to a change in the structure of normalized terms of degree four in $y_{1}, y_{2}, Y_{1}, Y_{2}$. They are given by Eq. (7.2).

For the resonance $\lambda_{1}+3 \lambda_{2}=0$ we have

$$
\begin{gathered}
c_{20}=\varepsilon^{2}\left[-\frac{1}{96}+O\left(\varepsilon^{2}\right)\right], \quad c_{11}=\varepsilon^{2}\left[-\frac{19+8 \sqrt{3}}{40}+O\left(\varepsilon^{2}\right)\right], \\
c_{02}=\varepsilon^{2}\left[-\frac{413+256 \sqrt{3}}{480}+O\left(\varepsilon^{2}\right)\right], \quad d=\frac{17843+9664 \sqrt{3}}{57600} \simeq 0.600374,
\end{gathered}
$$

and for the resonance $3 \lambda_{1}+\lambda_{2}=0$

$$
\begin{aligned}
& c_{20}=\varepsilon^{2}\left[-\frac{1}{96}+O\left(\varepsilon^{2}\right)\right], \quad c_{11}=\varepsilon^{2}\left[\frac{113+8 \sqrt{7}}{7560}+O\left(\varepsilon^{2}\right)\right], \\
& c_{02}=\varepsilon^{2}\left[-\frac{3347+512 \sqrt{7}}{30240}+O\left(\varepsilon^{2}\right)\right], \quad d=-\frac{1001437+154048 \sqrt{7}}{228614400} \simeq-0.006163 .
\end{aligned}
$$

In both resonant cases, both formal stability and stability for most initial conditions take place.

\section{Conclusion}

The above analysis shows that the resonant periodic motion of the pendulums with small values of the amplitude of horizontal oscillations of their suspension points is Lyapunov unstable if the dimensionless parameter $s$, which characterizes the stiffness of the spring connecting the pendulums, lies in the interval $1 / 4<s<3 / 4$.

In the interval $0<s<1 / 4$ the motion is stable for most (in the sense of Lebesgue measure) initial conditions and is also formally stable.

If $s>3 / 4$, formal stability takes place for all $s$ except for one value, $s=s_{4}=1 / 2+$ $+\sqrt{7} / 8 \simeq 1.401388$, which corresponds to the third-order resonance; for this value of $s$ the motion under study is Lyapunov unstable. If $s>3 / 4$ and $s \neq s_{4}, s \neq s_{*}=1.004417$, then the motion is stable for most initial conditions.

The stability at the boundary points of the intervals (5.2) and at the point $s=1$, which lies inside the second interval, has not been investigated. We note that, when $s=0$, we arrive at the problem of the stability of resonant periodic oscillations of one mathematical pendulum under horizontal oscillations of its suspension point; it is well known (see the monograph [50, Chapter 4]) that these oscillations are Lyapunov stable. The problem of the stability of the above-mentioned periodic motions of coupled pendulums in the cases $s=1 / 4,3 / 4$ and 1 are a subject for a separate study. 


\section{Conflict of Interest}

The authors declare that they have no conflict of interest.

\section{References}

[1] Markeev, A. P., Nonlinear Oscillations of Sympathetic Pendulums, Nelin. Dinam., 2010, vol. 6, no. 3, pp. 605-621 (Russian).

[2] Markeev, A. P., On the Stability of Nonlinear Vibrations of Coupled Pendulums, Mech. Solids, 2013, vol. 48, no. 4, pp. 370-379; see also: Izv. Akad. Nauk. Mekh. Tverd. Tela, 2013, no. 4, pp. 20-30.

[3] Markeev, A.P., A Motion of Connected Pendulums, Nelin. Dinam., 2013, vol.9, no.1, pp. 27-38 (Russian).

[4] Markeev, A.P. and Chekhovskaya, T. N., On Forced Oscillations of Sympathetic Pendulums, in 46th All-Russian Conf. on Problems of Mathematics, Computer Science, Physics and Chemistry. Section of Mathematics and Informatics (RUDN, Moscow, Apr 2010): Thesis of Reports, pp. 154156 (Russian).

[5] Malkin, I. G., Theory of Stability of Motion, Ann Arbor, Mich.: Univ. of Michigan, 1958.

[6] Malkin, I. G., Some Problems in the Theory of Nonlinear Oscillations: In 2 Vols., Germantown, Md.: United States Atomic Energy Commission, Technical Information Service, 1959.

[7] Birkhoff, G. D., Dynamical Systems, AMS Coll. Publ., vol. 9, Providence, RI: AMS, 1966.

[8] Giacaglia, G. E. O., Perturbation Methods in Non-Linear Systems, Appl. Math. Sci., vol.8, New York: Springer, 1972.

[9] Arnol'd, V.I., Kozlov, V.V., and Neřshtadt, A.I., Mathematical Aspects of Classical and Celestial Mechanics, 3rd ed., Encyclopaedia Math. Sci., vol. 3, Berlin: Springer, 2006.

[10] Moser, J. K., Lectures on Hamiltonian Systems, Mem. Amer. Math. Soc., No.81, Providence, R.I.: AMS, 1968.

[11] Markeev, A. P., Libration Points in Celestial Mechanics and Space Dynamics, Moscow: Nauka, 1978 (Russian).

[12] Korteweg, D. J., Sur certaines vibrations d'ordre supérieur et d'intensité anormale — vibrations de relations, - dans les mécanismes à plusieurs degrés de liberté, Arch. Néerl. sci. exactes et natur. Sér. 2, 1898, vol. 1, pp. 229-260.

[13] Beth, H. I. E., Les oscillations autour d'une position dans le cas d'existence d'une relation linéaire simple entre les nombres vibratoires, Arch. Néerl. sci. exactes et natur. Sér. 2, 1910, vol. 15, pp. 246-283.

[14] Beth, H.I.E., Les oscillations autour d'une position dans le cas d'existence d'une relation linéaire simple entre les nombres vibratoires (suite), Arch. Néerl. sci. exactes et natur. Sér. 3A, 1912, vol.1, pp. 185-213.

[15] Markeyev, A.P., Non-Linear Oscillations of a 1: 1 Resonance Hamiltonian System, J. Appl. Math. Mech., 2011, vol. 75, no. 6, pp. 631-646; see also: Prikl. Mat. Mekh., 2011, vol. 75, no. 6, pp. 901-922.

[16] Hénon, M. and Heiles, C., The Applicability of the Third Integral of Motion: Some Numerical Experiments, Astronom. J., 1964, vol. 69, no. 1, pp. 73-79.

[17] Roels, J., An Extension to Resonant Cases of Liapunov's Theorem Concerning the Periodic Solutions near a Hamiltonian Equilibrium, J. Differential Equations, 1971, vol. 9, no. 2, pp. 300-324.

[18] Braun, M., On the Applicability of the Third Integral of Motion, J. Differential Equations, 1973, vol. 13, no. 2, pp. 300-318.

[19] Breiter, S. and Elipe, A., Pseudo-Oscillator with a Quartic Perturbation, Mech. Res. Comm., 2001, vol. 28, no. 2, pp. 119-126.

[20] Kholostova, O. V. and Safonov, A. I., Investigation of the Motions of an Autonomous Hamiltonian System at a $1: 1$ Resonance, Regul. Chaotic Dyn., 2017, vol.22, no. 7, pp. 792-807.

RUSSIAN JOURNAL OF NONLINEAR DYNAMICS, 2020, 16(4), 607-623 
[21] Markeyev, A. P., Non-Linear Oscillations of a Satellite at 1: 1: 1 Resonance, J. Appl. Math. Mech., 2012, vol. 76, no. 1, pp. 36-47; see also: Prikl. Mat. Mekh., 2012, vol. 76, no. 1, pp. 52-68.

[22] Markeev, A.P., On Nonlinear Resonant Oscillations of a Rigid Body Generated by Its Conical Precession, Russian J. Nonlinear Dyn., 2018, vol. 14, no. 4, pp. 503-518.

[23] Kholostova, O.V., On the Motions of One Near-Autonomous Hamiltonian System at a $1: 1: 1$ Resonance, Regul. Chaotic Dyn., 2019, vol. 24, no. 3, pp. 235-265.

[24] Bardin, B.S. and Maciejewski, A. J., Transcendental Case in Stability Problem of Hamiltonian System with Two Degrees of Freedom in Presence of First Order Resonance, Qual. Theory Dyn. Syst., 2013, vol. 12, no. 1, pp. 207-216.

[25] Kholostova, O. V., Motions of a Two-Degree-of-Freedom Hamiltonian System in the Presence of Multiple Third-Order Resonances, Nelin. Dinam., 2012, vol. 8, no. 2, pp. 267-288 (Russian).

[26] Kholostova, O. V., Stability of Triangular Libration Points in a Planar Restricted Elliptic Three Body Problem in Cases of Double Resonances, Int. J. Non Linear Mech., 2015, vol. 73, pp. 64-68.

[27] Kholostova, O. V., The Interaction of Resonances of the Third and Fourth Orders in a Hamiltonian Two-Degree-of-Freedom System, Nelin. Dinam., 2015, vol. 11, no. 4, pp. 671-683 (Russian).

[28] Safonov, A. I. and Kholostova, O. V., On the Periodic Motions of a Hamiltonian System in the Neighborhood of Unstable Equilibrium in the Presence of a Double Three-Order Resonance, Vestn. Udmurtsk. Univ. Mat. Mekh. Komp. Nauki, 2016, vol. 26, no. 3, pp. 418-438 (Russian).

[29] Kholostova, O. V., On Periodic Motions of a Nonautonomous Hamiltonian System in One Case of Multiple Parametric Resonance, Nelin. Dinam., 2017, vol. 13, no. 4, pp. 477-504 (Russian).

[30] Safonov, A. I. and Kholostova, O. V., On Periodic Motions of a Symmetrical Satellite in an Orbit with Small Eccentricity in the Case of Multiple Combinational Resonance of the Third and Fourth Orders, Vestn. Udmurtsk. Univ. Mat. Mekh. Komp. Nauki, 2018, vol.28, no.3, pp.373-394 (Russian).

[31] Kholostova, O. V., On Periodic Motions of a Nearly Autonomous Hamiltonian System in the Occurrence of Double Parametric Resonance, Mech. Solids, 2019, vol.54, no. 2, pp. 211-233; see also: Prikl. Mat. Mekh., 2019, vol. 83, no. 2, pp. 175-201.

[32] Kholostova, O. V., On Multiple Fourth-Order Resonances in a Nonautonomous Two-Degree-ofFreedom Hamiltonian System, Vestn. Udmurtsk. Univ. Mat. Mekh. Komp. Nauki, 2019, vol. 29, no. 2, pp. 275-294 (Russian).

[33] Markeev, A. P., Linear Hamiltonian Systems and Some Problems on Stability of Motion of a Satellite about Its Center of Mass, Izhevsk: R\&C Dynamics, Institute of Computer Science, 2009 (Russian).

[34] Kolmogorov, A. N., Preservation of Conditionally Periodic Movements with Small Change in the Hamilton Function, in Stochastic Behaviour in Classical and Quantum Hamiltonian Systems (Volta Memorial Conference, Como, 1977), G. Casati, J. Ford (Eds.), Lect. Notes Phys. Monogr., vol. 93, Berlin: Springer, 1979, pp.51-56; see also: Dokl. Akad. Nauk SSSR (N.S.), 1954, vol.98, pp. 527-530.

[35] Arnol'd, V. I., Proof of a Theorem of A. N. Kolmogorov on the Invariance of Quasi-Periodic Motions under Small Perturbations of the Hamiltonian, Russian Math. Surveys, 1963, vol. 18, no. 5, pp. 9-36; see also: Uspekhi Mat. Nauk, 1963, vol. 18, no. 5, pp. 13-40.

[36] Arnold, V. I., Small Denominators and Problems of Stability of Motion in Classical and Celestial Mechanics, Russian Math. Surveys, 1963, vol.18, no.6, pp.85-191; see also: Uspekhi Mat. Nauk, 1963, vol. 18, no. 6, pp. 91-192.

[37] Arnol'd, V.I., Mathematical Methods of Classical Mechanics, 2nd ed., Grad. Texts in Math., vol. 60, New York: Springer, 1997.

[38] Nekhoroshev, N. N., Behavior of Hamiltonian Systems Close to Integrable, Funct. Anal. Appl., 1971, vol. 5, no. 4, pp. 338-339; see also: Funkts. Anal. Prilozh., 1971, vol. 5, no. 4, pp. 82-83.

[39] Nekhoroshev, N. N., An Exponential Estimate of the Stability Time of Near-Integrable Hamiltonian Systems, Russian Math. Surveys, 1977, vol.32, no.6, pp.1-65; see also: Uspekhi Mat. Nauk, 1977, vol. 32 , no. $6(198)$, pp. 5-66. 
[40] Nekhoroshev, N. N., An Exponential Estimate of the Time of Stability of Nearly Integrable Hamiltonian Systems: 2, in Topics in Modern Mathematics, O. A. Oleinik (Ed.), Petrovskii Semin., no. 5, New York: Consultant Bureau, 1985, pp.1-58; see also: Tr. Semin. Petrovsk., 1979, no. 5, pp. 5-50.

[41] Moser, J., New Aspects in the Theory of Stability of Hamiltonian Systems, Comm. Pure Appl. Math., 1958, vol.11, no. 1, pp. 81-114.

[42] Moser, J., Stabilitätsverhalten kanonischer Differentialgleichungssysteme, Nachr. Akad. Wiss. Göttingen. Math.-Phys. Kl. IIa, 1955, vol. 1955, pp. 87-120.

[43] Moser, J., On the Elimination of the Irrationality Condition and Birkhoff's Concept of Complete Stability, Bol. Soc. Mat. Mexicana (2), 1960, vol. 5, pp. 167-175.

[44] Moser, J., Stability of the Asteroids, Astron. J., 1958, vol.63, no. 10, pp. 439-443.

[45] Siegel C. L. Vorlesungen über Himmelsmechanik, Grundlehren Math. Wiss., vol. 85, Berlin: Springer, 1956.

[46] Glimm, J., Formal Stability of Hamiltonian Systems, Comm. Pure Appl. Math., 1964, vol. 17, no. 4, pp. 509-526.

[47] Bryuno, A.D., Formal Stability of Hamiltonian Systems, Math. Notes, 1967, vol.1, no.3, pp. 216-219; see also: Mat. Zametki, 1967, vol. 1, no. 3, pp. 325-330.

[48] Bruno, A. D., The Restricted 3-Body Problem: Plane Periodic Orbits, de Gruyter Exp. Math., vol. 17, Berlin: Walter de Gruyter \& Co., 1994.

[49] Gel'fond, A. O., Solving Equations in Integers, Mineola, N.Y.: Dover, 2018.

[50] Kholostova, O. V., Problems of Dynamics of Solids with Vibrating Suspension, Izhevsk: R\&C Dynamics, Institute of Computer Science, 2016 (Russian). 